\title{
NOTAS SOBRE LA GÉNESIS FENOMENOLÓGICA DEL CONCEPTO LEVINASIANO DE DIACRONÍA.
}

\section{Gisela Suazo*}

\section{Introducción.}

La propuesta levinasiana de la noción de diacronía, introducida por el autor en 1946 en Le temps et l'Autre, describe la instancia en la que se cumple la relación con lo absolutamente otro, es decir, con la alteridad en sentido estricto. La diferencia irreductible de la alteridad respecto de las estructuras cognitivas del Mismo se cumple en el acontecimiento del rostro del otro, cuya irrupción interrumpe la continuidad del fenómeno originando el modo específico de manifestación suprafenomenológico de la revelación. La revelación anuncia la manifestación de una realidad que permanece no obstante escondida, inasible como tal por la sensibilidad receptiva y por la inteligencia humanas. La revelación ofrece ver lo invisible, pero no como visibilidad plena sino como retirada de aquello que se da. Esto ocurre a contracorriente del gesto propio de la fenomenología que por medio del logos satura lo visible ocultando la retirada y desconociendo que la altura de la trascendencia reside en la manifestación de lo que permanece oculto. La diacronía es la relación con dicha invisibilidad.

El término "diacronía" mienta esta diferencia infinita que abstrae la alteridad de cualquier intento de asunción cognoscente, sea en el nivel de la conciencia meramente receptiva, sea en el nivel de la reproducción conceptual. Autrement qu'être ou au-delà de l'essence avanza en una sistematización de la noción de diacronía a partir de un diálogo permanente

\footnotetext{
* Investigadora-Docente de la Universidad Nacional de General Sarmiento. Forma parte de los equipos docentes a cargo de las asignaturas de Gnoseología y Antropología Filosófica. Fue becaria del CONICET y actualmente escribe su tesis doctoral sobre la fenomenología del tiempo y de la historia en Emmanuel Lévinas. Dirección electrónica: gsuazo@ungs.edu.ar
} 
con las concepciones formales de tiempo como la que presentan la estética trascendental kantiana, la noción de "flujo de la conciencia" en Husserl y la concepción hegeliana del tiempo como forma del desenvolvimiento dialéctico del contenido de la Idea. En todos los casos, explica Lévinas, las filosofías de la temporalidad se ocupan de desentrañar la forma misma de la temporalidad sin exigir una condición, es decir, "algún sustrato de 'materia' o de acontecimiento, un contenido sentido de alguna manera previo a la forma"1. Discrepando con estas posiciones, Lévinas se mantiene en el terreno de la experiencia ética como el dominio correcto para comprender el sentido originario del tiempo, vale decir: el tiempo como trascendencia. A lo largo de su obra (desde 1935 en adelante) se registran diversas afecciones o estados del sujeto que componen la trama de la experiencia del tiempo: la náusea, la vergüenza, el dolor, el cansancio, la pereza, el hastío, el insomnio, la paciencia, la esperanza, la muerte, la responsabiliāad por el otro, etc. La hermenéutica que desarrolla sobre cada una de esas modalidades de la existencia confluye finalmente en la formulación del proyecto de nuestro autor de desformalizar (déformaliser) la noción de tiempo ${ }^{2}$, propósito que reclamaría el reconocimiento de una situación empírica privilegiada en función de la cual sean finalmente comprensibles los modos de desvanecimiento en el pasado y de inminencia del futuro.

El proceso de desformalización de la noción del tiempo está presente desde los primeros análisis de Lévinas, aunque tal proyecto no haya sido formulado en esos términos hasta en sus últimos trabajos, que datan de la década del '80, como es el caso. de Diachronie et representation, publicado en el año 1985 en la Revue de l'Université d'Ottawa. En tal sentido, Lévinas admite, en el prefacio de 1979 a Le temps et l'Autre, la originalidad con la que tempranamente se había ya planteado la cuestión del tiempo, esto es, el tiempo no como el horizonte ontológico del ser y del ente sino como relación del pensamiento con lo totalmente otro, relación que se cumple en las situaciones concretas de socialidad en las que nos encontramos frente al rostro del otro en cuanto rostro; sentido del tiempo al que Lévinas le atribuye el nombre de diacronía. Si bien la noción de diacronía ha sido

${ }^{1}$ Emmanuel Lévinas, Entre Nous. Essais sur le penser-à-l'autre, Paris, Livre de Poche, 1993. p. 263. Esta obra se citará con la sigla EN.

2 Ibidem. 
desarrollada a partir del acontecimiento de la relación de proximidad, creemos -y ésta es nuestra hipótesis de trabajo aquí- que la nota central que hace a su carácter refractario respecto del régimen intencional de la conciencia $\mathrm{y}$, por tanto, anárquico respecto del presente de la representación, están en cierta forma iluminados en Lévinas a partir de su lectura crítica de las Lecciones sobre la fenomenología del tiempo interno de Edmund Husserl, más precisamente de la noción de impresión originaria (Urimpression) allí expuesta.

El presente artículo tiene como objetivo principal, entonces, exponer la génesis de la noción de tiempo diacrónico. Para alcanzar este objetivo planteamos el siguiente recorrido: en primer lugar, repasar muy sucintamente el sentido que asume la diacronía en Le temps et l'Autre, que es el primer texto (según el orden cronológico) en el que se formula dicha categoría, para referirnos más detenidamente a lo que consideramos la matriz fenomenológica de la misma. En este sentido, nos proponemos en segundo lugar, indagar la recuperación crítica de la figura fenomenológica de Urimpression que realiza Lévinas especialmente en Autrement qu'être ou audelà de l'essence (en el parágrafo sobre "Vivencia sensible") y en el artículo de 1965 "Intentionalité et sensation", con la finalidad de indicar la significación del presente absoluto de la impresión originaria como "presente diacrónico".

\section{La diacronía del tiempo como relación con la trascendencia: Le temps et l'Autre (1949).}

Le temps et l'Autre introduce explícitamente una nueva concepción del tiempo como trascendencia que - advierte el propio autor en el prólogo de 1979 - constituye "el nacimiento y la formulación primera"3 del proyecto principal de su filosofía. Las conferencias de 1946, reunidas en ese volumen, tuvieron el propósito de cuestionar la preeminencia del tiempo concebido como el horizonte ontológico del ser y del ente, y exponer su tesis central sobre la producción del tiempo a partir de la relación interhumana.

${ }^{3}$ Emmanuel Lévinas, Le temps et l'Autre, Montpellier, Fata Morgana, 1979, p. 9. Esta obra se citará con la sigla $T A$. 
La citada obra esboza la concepción del tiempo como la materialidad de una relación que se caracteriza por la imposibilidad de coincidir con el otro término del vínculo. El tiempo se produce en el seno de una relación donde el Otro es tal en tanto que invisible y refractario a la razón. Es el insoslayable fenómeno de la no-coincidencia lo que caracteriza la tesis original sobre el tiempo en Lévinas y recibe el nombre de "diacronía": "Imposibilidad de coincidir, in-adecuación, que no son nociones simplemente negativas, sino que tienen un sentido en el fenómeno de la nocoincidencia dado en la dia-cronía del tiempo"4.

De acuerdo con Lévinas, en la relación del pensamiento con aquello que se manifiesta inconmensurable con su capacidad de aprehensión pero que, no obstante, conserva con él un lazo de proximidad irreductible, anida el sentido originario del tiempo. La verdadera alteridad trasciende la orquesta de posibilidades cognitivas del yo-pienso guardando su diferencia a distancia de todo concepto, categoría, idea o representación. Así, la tesis original sobre el tiempo implica la realización de un acontecimiento sin aprehensión ni representación; un suceso que, vale decir, se cumple más allá de las estructuras que ordenan atemporalmente el ente en el todo del ser.

En la relación social y ética que Lévinas insiste en llamar "relación cara a cara", el otro hombre no lleva su otredad como un atributo más desprendido de un juicio de relación. Muy por el contrario, la alteridad es el contenido mismo del hombre y es lo que hace de ese otro, no "otra cosa" sino, justamente, "otro hombre".

En la relación interpersonal la expresión del rostro del otro señala una negación a los poderes del yo-pienso y en este sentido su epifanía implica una "resistencia total a la aprehensión"5. Ahora bien, la resistencia del rostro no disputa el alcance de las facultades cognitivas del yo pienso, sino que interpela "mi poder de poder"6. El Rostro del Otro se expresa y se resiste a la actividad pretendidamente hegemónica del yo pienso sin oponer fuerza alguna. Lo hace exponiendo "en la desnudez de sus ojos" y en la

${ }^{4}$ TA, p. 10.

5 Emmanuel Lévinas, Totalidad e infinito. Ensayo sobre la exterioridad, Salamanca, Sígueme, 1987, p. 211. Se citará con la sigla TI.

${ }^{6}$ Ibidem. 
vulnerabilidad de su vida corporal una existencia totalmente ajena a la de las formas (llámense "conceptos", "categorías", "leyes" o "géneros"). La desnudez propia del rostro conmina a la comprensión de su debilidad, carencia y peligro mucho antes de devolverlo a la imagen como un objeto contabilizable entre los bienes y saberes acopiados. La alteridad tiene su lugar de exposición en el rostro, y "manifestarse como rostro -como se aclara en $T I$ - es imponerse más allá de la forma, manifestada como puramente fenomenal, presentarse de una manera irreductible a la manifestación, como la rectitud del cara a cara, sin la mediación de la imagen en su desnudez, es decir, en su miseria y en su hambre"”.

La relación con lo totalmente Otro es relación con una trascendencia que no puede sernos indiferente pero que, a su vez, entra en la relación sin comprometer su alteridad. Relación con lo que queda afuera, es decir, con lo que no es alcanzado por el ejercicio de síntesis en la representación. "Quedar afuera" significa aquí mantenerse en un plano diferente al de la representación, significa entonces, también, una realidad que no toma nada de los procesos de constitución de la conciencia. El sentido genuino del tiempo reside, según Lévinas, en esta relación con lo inasimilable, con lo que está más allá de nuestras posibilidades de comprehensión y reducción.

A continuación intentaremos exponer en qué sentido esta noción de diacronía recoge elementos centrales para su determinación de una lectura crítica que hace Lévinas de las Lecciones de fenomenología de la conciencia del tiempo inmanente de Husserl centrándose especialmente en la categoría de Urimpression.

\section{El influjo de la lectura levinasiana de Husserl en la construcción de la categoría de diacronía.}

La hipótesis que sostiene nuestra lectura es que la fenomenología levinasiana sobre el tiempo diacrónico descansa en una interpretación crítica y fecunda de la noción husserliana de 'presente viviente' (en el que tiene lugar la impresión originaria o Urimpression) en tanto le permite a nuestro autor dar cuenta de una producción originaria del tiempo a través de un

$7 T 1$, p. 213. 
desplazamiento fuera del dominio del pensamiento y del presente de la representación. Por tanto, trataré aquí de describir en qué sentido Lévinas encuentra en la función gnoseológica de la Ur-impression husserliana, por una parte, un elemento ajeno a la intencionalidad constituyente que caracteriza a la conciencia trascendental de la fenomenología husserliana y, por otra, un elemento descriptivo de un acontecimiento temporal sin génesis en ni reducción al decurso del tiempo interno en el cual se ordena el conjunto de nuestras vivencias y se sintetiza la identidad del ente.

Con este análisis pretendemos también destacar que la concepción levinasiana del tiempo guarda una relación no sólo de recusación de la fenomenología husserliana, sino también de recuperación fructífera de la categoría de Urimpression para la construcción de una idea del tiempo como trascendencia o como apertura hacia la alteridad.

Ciertamente, para Lévinas lo sensible no se reduce a la función cognoscente de la receptividad, y su pasividad radical no se limita tampoco a la conciencia intuitiva, sino que señala un ámbito absolutamente refractario a la conciencia y a su temporalidad. No obstante, aunque refractaria, la sensibilidad ofrece -según los textos levinasianos que consultamos- una suerte de condición de posibilidad de la conciencia originaria del tiempo y de la conciencia a secas. Este modo de comprender lo propio de la sensibilidad como una dimensión heterogénea pero creadora de la actividad de la conciencia y el proceso de conocimiento parece encontrar en la fenomenología husserliana elementos que le sirven de precedentes conceptuales.

Lévinas realiza una lectura de la Fenomenología de la conciencia del tiempo interno que enfatiza la índole originaria de la sensibilidad y que le permite mostrar que incluso en los análisis husserlianos el sentir constituye el punto de partida del tiempo y de la vitalidad de la conciencia. La peculiaridad de la interpretación levinasiana de las Lecciones está en radicalizar el sentido de originariedad que distingue a las impresiones en tanto Ur-impression respecto de otros modos de conciencia. A través de esta lectura, el autor aspira a mostrar que en el nivel de las sensaciones opera una dimensión temporal que excede su análisis en el marco de una conciencia estrictamente intencional. Nos interesa aquí establecer nexos entre esta lectura crítica y la noción levinasiana del tiempo "trans-intencional" de la diacronía. 
Sin intención de agotar este aspecto de la aproximación levinasiana a la fenomenología husserliana, advertimos que existen por lo menos dos lugares en los que nuestro autor expone las claves de su comprensión de la noción de Urimpression que aquí nos importa recuperar: "Intentionalité et sensation" (texto del año 1965), recopilado más tarde (1967) en En découvrant l'existence avec Husserl et Heidegger, y Autrement qu'être. La exposición de esta cuestión aparece, en lo que sigue, dividida en tres partes que corresponden a una breve descripción de tres instancias argumentativas que, según entiendo, articulan la lectura levinasiana de las Lecciones de Husserl. Dichas instancias son, en primer lugar, el reconocimiento de la sensibilidad como fuente originaria del tiempo. En segundo lugar, el carácter peculiar de la retención en tanto condición de posibilidad del fenómeno temporal y en tanto modalidad en la que se produce la temporalización. En tercer lugar, la problematicidad que sugiere la noción husserliana de "conciencia impresional" y su vínculo con la concepción de tiempo diacrónico en Lévinas.

\subsection{La sensibilidad como fuente originaria del tiempo.}

El primer elemento que ofrece Lévinas a favor de la tesis sobre el antecedente fenomenológico de la concepción que afirma que el tiempo interno se temporaliza a partir de un dato de la sensibilidad, se halla en la sentencia del propio Husserl según ìa cual la "sensación es aquello que tomamos como conciencia originaria del tiempo"s. De acuerdo con este autor, el tener sensaciones es constitutivo de la conciencia originaria del tiempo, puesto que el carácter temporal de la conciencia que acompaña a toda impresión es "presentativo", vale decir que es la presentación de un contenido intuitivo con el carácter de abora (vinculado a la presentación retencional con el carácter de recién sido). La conciencia de la sensación (o conciencia impresional) se desenvuelve presentando siempre el nuevo ahora cuyo contenido primario podrá ser retenido y posteriormente conceptualmente determinado pero no extraído del propio flujo temporal de la conciencia.

${ }^{8}$ Edmund Husserl, Fenomenología de la conciencia del tiempo inmanente, trad. de Otto E. Langfelder, Buenos Aires, Nova, 1959, Anexo III, p. 162. Esta obra se citará con la sigla LFT. 
Los actos de recuerdo, espera y fantasía introducen modificaciones en este material. Husserl señala tres modos esenciales de la conciencia temporal: por un lado, el de la actualización posicional (que se precisa bajos los modos del recuerdo y de la espera); por otro, el de la actualización de la fantasía, y, finalmente, la "sensación" como "esfera originaria" en cuanto presentación de los contenidos primarios que introducen la novedad del ahora. Los modos de la conciencia temporal presentativa son el de la presentación con el carácter de ahora y, ligada a ésta, aquella con el carácter de "recién sido"; ambas pertenecen a la conciencia presentativa concreta (que nombramos ordinariamente como "presente"). La conciencia impresional introduce el ahora que activa y da contenido a la conciencia retencional y con ello también a la posibilidad de la representación en general y del recuerdo secundario. La producción de la conciencia constituyente de los objetos gracias a sus determinaciones temporales es tributaria de este nivel de conciencia originario, pues tiene su inicio en la sensación.

\subsection{El carácter "peculiar" de la retención.}

La intencionalidad a partir de la impresión sensible contiene las intencionalidades temporales que inauguran el horizonte primario del tiempo con orientación hacia el pasado y el futuro: conciencia retencional y conciencia protencional. Para indicar brevemente en qué consisten cada una de las mencionadas intencionalidades, diremos que ambas forman parre del horizonte viviente del ahora y constituyen modificaciones de la fase impresional. Las protenciones son intenciones anticipatorias y las retenciones son intenciones orientadas hacia lo anteriormente percibido. Las dos clases de intuiciones están contenidas en la percepción del objeto. Concretamente, podemos decir que en la percepción se distinguen tres niveles. En primer lugar, el nivel de la protoimpresión o de la impresión (que comporta el grado de intuitividad más elevado y es el modo de darse de cualquier objeto con originalidad) que tiene como correlato el momento temporal del "ahora". En segundo lugar, la intencionalidad retencional y protencional está presente en cada impresión porque junto a cada fase impresional que me da el ahora "en carne y hueso" del objeto, se me ofrecen también retenciones de las fases pasadas de la manifestación del 
objeto así como también intenciones anticipativas - protenciones- de sus fases futuras.

Centrándose en el análisis del valor de la retención en la economía de la teoría del tiempo husserliana, Lévinas afirma que la "intencionalidad peculiar" de la retención es ni más ni menos que el propio tiempo?. A continuación intentamos exponer brevemente los argumentos que sostienen esta lectura de la intencionalidad retencional.

Según las Lecciones $^{10}$, la retención consiste en un decrecimiento de los contenidos impresionales, es decir que la conciencia impresional se transforma a través de un constante fluir en una conciencia retencional. Cuando una percepción pasa a la retención su contenido empalidece y esto sucede porque dicho contenido ya no está temporalmente presente (en sentido estricto), sino justamente recordado de un modo primario en el ahora. Así, cada ahora actual está, por un lado, subordinado a esta ley de modificación según la cual toda conciencia impresional cambia constantemente en un 'ha sido' y, por otro lado, cada "ahora" ulterior constituye también una retención de cada punto anterior. La retención permite conservar conciencia de la fase antecedente del objeto, esto es, mantener "como si" (aún) estuviera presente la impresión del ahora "recién sido" asociada al contenido del ahora actual. Así, la retención hace posible la retrospección sobre lo transcurrido, esto es, la reflexión, la objetivación y la rememoración.

La modificación retencional es, además, continua, esto es, “cobija, diremos, en forma de una serie perspectivista, el legado del pasado"11. En consecuencia, hablar de retención no refiere únicamente a la modificación constante que emerge de la protoimpresión sino a una corriente de modificación continua del mismo puntc. El contenido de la conciencia impresional es conservado por el continuo retencional a través de una serie

${ }^{9}$ Cfr. Emmanuel Lévinas, Autrement qu'être ou au-delà de l'essense, The Haye, Martinus Nijhoff, 1978. Se utilizará la edición española: Emmanuel Lévinas, De otro modo que ser o más allá de la esencia, trad. de Antonio Pintor Ramos, Salamanca, Sígueme, 1987, p. 80 (Esta obra se citará con la sigla DMS).

${ }^{10}$ Cfr. LFT, parágrafo 12 , pp. 79-80.

${ }^{11}$ LFT, p. 77. 
de modificaciones que describe el trayecto del punto inicial de la protoimpresión en un ahora actual hacia el trasfondo indiferenciado del pasado.

Cabe aclarar que la retención no es un acto que convierta por sí mismo la fase transcurrida en objeto, sino que permite mantenerla "en el puño"12 para dirigir luego la mirada sobre ella. La conciencia retencional no es un acto sino una "conciencia momentánea de la fase transcurrida"13.

Lévinas subraya la preeminencia de la retención en la concepción husserliana del tiempo. Advierte que la peculiaridad de la intencionalidad retencional es el concepto nuclear de la estructura del tiempo ${ }^{14}$. Según Lévinas, la intencionalidad retencional y la intencionalidad protencional que retienen o anticipan la identidad de la sensación y acompañan el instante de la conciencia impresional, constituyen la conciencia misma del tiempo ${ }^{15}$. Estas intencionalidades describen la conciencia de lo que ha sido en un pasado inmediato y de lo que se anticipa para un futuro también inmediato. La conciencia del dato impresional con el índice de "recién sido" o con el índice de "aún no" miden y llevan a cabo la distancia entre el instante de la proto-impresión actual y el de la proto-impresión anterior. Estos actos de mención son conciencia de los horizontes primarios del tiempo pasado y futuro y -a la vez- instituyen las fases temporales de la percepción - pasado y futuro. En consecuencia, más allá de la condición necesaria que la intencionalidad retencional comporta para la articulación de la representación objetiva, la retención cumple, desde la perspectiva levinasiana, la obra misma del tiempo en tanto ella coincide con el acontecimiento de la distancia temporal. En otras palabras, es la conciencia retencional de una protoimpresión que ya no ocupa el lugar de puntofontanal, mentada como recién transcurrida pero aún resonando en el presente, donde reside el acontecimiento del tiempo, es decir, donde tiene

\footnotetext{
${ }^{12}$ Cfr. LFT, p. 181. La expresión es de Husserl.

13 Ibidem.

${ }^{14}$ Cfr., DMS, p.80.

${ }^{15}$ Cfr. Emmanuel Lévinas, Descubriendo la Existencia con Husserly con Heidegger, trad. de Manuel Vázquez, Madrid, Síntesis, 2005, p. 220. Esta obra se citará con la sigla DEHH.
} 
lugar la conciencia de la distancia entre la nueva proto-impresión y la primera proto-impresión retenida (conceptualmente) en la segunda.

Según la lectura de Lévinas, la conciencia de esta distancia, la retención, es la producción misma de esa distancia que debe comprenderse como distancia temporal. En este sentido, el filósofo afirma: "La distanciación es retención y la retención es distanciación: la conciencia del tiempo es el tiempo de la conciencia"16. En Autrement qu'être, nuestro autor emplea la expresión "diástasis de lo puntual"17 para referirse al desplazamiento de una protoimpresión hacia las fases retencionales de la conciencia, produciendo la "distancia temporal"18.

Desde esta perspectiva, el tiempo no aparece como una estructura autárquica capaz de recibir sensaciones y de disponerlas en un decurso previamente establecido, sino como un ejercicio de distanciación que se despliega originalmente a partir de una Urimpression desplazada hacia atrás por la llegada de una Urimpression nueva. Esta distanciación es la retención misma y constituye una especie de "demora" en la toma de conciencia de un dato que pasa de la ambivalencia del ahora presente, pero invisible, a la visibilidad del pasado retencional con el índice de "ya no es".

La distanciación entre una Urimpression y otra es el acontecimiento primordial del flujo temporal. La medición de ese desfase coincide con ia conciencia del mismo y describe la temporalización misma del tiempo interno. Desde esta perspectiva, Lévinas afirma que "la mirada intencional que constata la distanciación es esta distanciación misma"19. La conciencia interna del tiempo, anunciada en el título de las Lecciones, no consiste en una reflexión sobre el tiempo sino en la producción misma del tiempo: la temporalización. Desde esta lectura expuesta en "Intentionalité et sensation" comprendemos la afirmación hecha más tarde por Lévinas en Autrement qu'être de que "esta intencionalidad específica [de la retención] es el propio tiempo"20.

\footnotetext{
${ }^{16} \mathrm{DEHH}$, p. 222.

${ }_{17} \mathrm{DMS}, \mathrm{p} .81$.

18 Cfr. DEHH, pp. 218-222.

${ }^{19} \mathrm{DEHH}$, p. 221.

${ }^{20} D M S$, p. 80.
} 
Aunque el propio Lévinas pone el acento en recordar la función cardinal de la retención, su lectura de las Lecciones subraya sobre todo el carácter primordial de la impresión originaria sobre el conjunto del decurso de la temporalidad inmanente.

\subsection{La originariedad de la protoimpresión.}

Lévinas destaca también que la retención "es impensable sin un correlato aprehendido" 21 . En efecto, el propio Husserl, cuando analiza el continuo constituyente de tiempo como una corriente de constante producción de modificaciones de modificaciones, indica que el principio del que parte esa serie infinita de modificaciones - ese "punto cero" - es una protoimpresión o impresión originaria fundadora de una nueva fase de la duración concreta y que indefectiblemente se sumergirá en un decrecimiento de cambios a un ritmo proporcional al ingreso de nuevas protoimpresiones $^{22}$. Cada protoimpresión agrega un nuevo ahora a la vez que modifica el ahora anterior en un "no ahora". En relación con este punto Husserl dice que esta agregación de "ahoras" es ordinariamente mentada a través de expresiones como "producción" o, incluso, "nacimiento repentino de una fuente". Sin embargo, estas palabras no dejan de tener una alusión metafórica en relación con la generación de cada punto ahora de la duración concreta del tiempo, pues "no son algo producido por la conciencia, sino lo protogenerado, lo 'nuevo', lo que ha nacido de una manera extraña a la conciencia"23.

Cada impresión originaria marca siempre el comienzo de una producción de sucesivas modificaciones y asociaciones que se traducirán en las distintas fases temporales del objeto. La impresión originaria es por eso también fuente originaria, puesto que significa el punto de partida desde el cual se lleva a cabo la producción constante de modificaciones retencionales y asociaciones, "mas ella misma [la protoimpresión] no es producida, no se origina como producto sino mediante una genesis spontanea: es

\footnotetext{
${ }^{21}$ Ibidem, p. 80.

${ }^{22}$ Cfr. LFT , Anexo 1, pp. 151-153.

${ }^{23} L F T$, p.153.
} 
protogeneración"24. Nuestro autor lo enfatiza: ella "no brota, (no tiene germen) sino que es protocreación" 25 . La protofuente a partir de la cual tiene lugar el trabajo conciencial de hacer evolucionar y crecer lo protogenerado emerge más allá del devenir del flujo temporal de la conciencia. La producción de la conciencia descansa inevitablemente en la pasividad de la recepción de la protoimpresión y es en este sentido que Husserl afirma que "lo único que cabe decir es que la conciencia no es nada sin la impresión”26.

El elemento condicionante que hay que añadir a la dinámica retencional del tiempo interno es que sin impresión alguna no hay "ahora" válido como fuente de la cual broten las modificaciones retencionales ni novedad que promueva el desplazamiento de cada ahora a un "no ahora" retenido "conceptualmente". Teniendo en cuenta que la producción de la conciencia procede siempre a partir de los datos recibidos de las sensaciones o impresiones hacia las correlativas retenciones e interrelaciones de tales modificaciones, Lévinas encuentra en la protoimpresión el vértice donde nace la dinámica del tiempo y la conciencia en tanto corriente de vivencias, y asegura entonces que "tiempo, impresión sensible y conciencia se conjugan"27.

\subsection{La problematicidad de la conciencia impresional.}

El tercer elemento crítico en la lectura levinasiana de las Lecciones se refiere a cierta problematicidad que sugiere la conciencia impresional en tanto conciencia de un contenido que ella misma no puede proyectar y cuyo nacimiento es totalmente ajeno a su actividad productiva.

El nivel excepcionalmente originario de la proto-impresión comporta una conciencia no-intencional eximida de todas las características constitutivas de la "especificidad retencional". La proto-impresión o impresión originaria en el instante de su evento no recibe modificación retencional alguna y es, según lo ya expuesto, el punto inicial a partir del cual

\footnotetext{
${ }^{24}$ LFT, p.152.

${ }^{25}$ Ibidem.

26 IFT', p. 153.

27 DMS, p. 80.
} 
resulta factible la propia corriente del continuo retencional. Husserl es en este sentido taxativo cuando afirma que "la proto-impresión es absolutamente inmodificada, la fuente primigenia de toda otra conciencia y de todo ser"'28, es decir, reconoce que ella no es un producto de la conciencia aunque sí constituye un principio absoluto para toda su producción.

No obstante, aunque la proto-impresión transcurra a un nivel tan originario al que ni siquiera le es aplicable el carácter intencional "en el sentido 'específico' de la retención"29 "la proto-impresión no se imprime sin conciencia"30. El padre de la fenomenología responde, entonces, sobre el espesor fenomenológico de la proto-impresión, pues dado que ésta se produce sin modificación retencional, cabe la posibilidad de pensarla como un acontecimiento inconsciente. Contra esto último Husserl alega que aunque la proto-impresión no comporta una conciencia objetal la cual exigiría no sólo la labor de la retención sino también la reflexión o reproducción) implica sin embargo un tipo de conciencia, puesto que la conciencia es por definición "ser consciente" activo en cada una de sus fases. Por otra parte, advierte que si la conciencia sólo se lograse por la retención se volvería incomprensible el modo en el que la fase inicial alcanza a revestir el carácter de "ahora". Ciertamente, la conciencia de una impresión originaria que se cumple bajo la modalidad temporal del ahora es el fundamento de toda retención, pues "una retención de un contenido inconsciente es imposible" 31 . Además, sería absurdo que un acontecimiento que se cumple de un modo inconsciente sea intencionado con el índice de ahora-recién-sido.

De acuerdo con Husserl, la clave para evitar estas contradicciones estaría en no tomar esta proto-conciencia que tiene lugar en la Urimpression como un acto aprehensivo, sino como una conciencia pre-objetal en la que ésta contiene la impresión sin hacerla objeto. El acto aprehensivo de la

${ }^{28}$ LFT, p. 116.

${ }^{29}$ DMS, p. 80. Especificidad que consiste en la conservación "en el concepto" de una fase antecedente, en virtud de lo cual es factible la retrospección sobre lo transcurrido.

${ }^{30} \mathrm{DMS}$, p. 81.

${ }^{31}$ LFT, p. 181. 
reflexión que vuelve la mirada sobre ella (en cuanto contenido) presupone todo el juego de la proto-conciencia y de las retenciones.

Lévinas muestra cómo una lectura sobre la noción husserliana de Urimpression que lleve hasta sus últimas consecuencias su condición de originariedad, haciendo hincapié en el carácter no objetal ni aprehensivo que supone la proto-conciencia, pone en tela de juicio la preeminencia de "la conciencia objetivadora y la hegemonía de la representación" 32 . En este sentido, afirma que "la protoimpresión encuentra de nuevo su poder de asombrar en el contexto de la intencionalidad"33.

Lévinas intenta extraer del planteo husserliano sobre el presente viviente en el que se realiza la protoimpression una oscuridad capaz de sugerir un nuevo orden temporal irreductible al de la conciencia, como la modalidad en la que se producen los acontecimientos sensibles. Este otro tiempo recibe el nombre de tiempo diacrónico, y se opone al tiempo de la conciencia, es decir, al tiempo de lo recuperable que garantiza que "nada pueda suceder al ser clandestinamente, que nada pueda desgarrar el hilo de la conciencia" 34 .

Ciertamente, en las Leccione se describe el advenimiento de la impresión como una genesis spontanea completamente ajena a la producción de la conciencia; sin embargo, inmediatamente se procede al análisis de su temporalización, mostrando cómo - a partir de la "variación en la identidad" que permite la retención - se torna lógicamente posible su rememoración y su reconstrucción en la identidad objetiva. El fllósofo lituano enfatiza en cambio la existencia de un elemento que escapa a la dinámica intencional de la conciencia y que, por tanto, no puede ser retenido. En efecto, en "Intentionalité et sensation" Lévinas explica que en Husserl la noción de sensación logra eximirse de la actividad constituyente e iluminadora de la intencionalidad. En el citado estudio, Lévinas hace hincapié en las propias afirmaciones de Husserl en las Lecciones (más precisamente en el Apéndice V) que ponen en cuestión el lugar primordial de la aprehensión animadora del dato impresional en el acto de la

\footnotetext{
${ }^{32}$ DMS, p. 81.

${ }^{33} \mathrm{DMS}$, p. 81.

34 DMS, p. 82.
} 
percepción, al preguntarse si dicha percepción comienza con el dato impresional, "o si éste no tiene que estar constituido antes de que pueda comenzar la aprehensión animadora"35. Al respecto, el autor de "Intentionalité et sensation", advierte que si bien el objeto constituido es posible por la mediación de una intención que anima la sensación, es necesario que esta sensación haya transcurrido al menos mínimamente y de manera autónoma para que -posteriormente- una intención sea capaz de "inspirarla"36. En consecuencia, "el acto es, pues, posterior al material constituido" 37 , razón por la cual la conciencia adquiere una estructura de "rezago" en el pasado retencional, en la medida en que la conciencia objetivadora aprehende diferidamente el material que se ofrece en el presente absoluto de la proto-impresión. Por esta razón, el presente viviente o punto temporal ahora en el que se desenvuelve la proto-impresión comporta un acontecimiento anárquico frente al ejercicio intencional de la conciencia.

Según Lévinas, los datos hyléticos o material sensible están en la base de la intencionalidad pero "lejos de desempeñar en el sistema un papel de residuo cuya evacuación progresiva debía propiciarse, la sensación ocupa en la meditación husserliana un lugar cada vez mayor"38.

Nuestra hipótesis de lectura es que en la noción husserliana de "presente viviente" Lévinas encuentra cierta oscuridad fecunda capaz de iluminar un sentido diferente del acontecimiento sensible y, con él, la sospecha del sentido diacrónico del tiempo. Dicha oscuridad estriba en que la noción de presente viviente trata de hacer inteligibles las nociones de origen y de creación caracterizándolas como una especie de espontaneidad en la que el límite entre la actividad y la pasividad de la conciencia no queda claro y donde ambas "se confunden de modo absoluto"39. Garrido Maturano ${ }^{40}$ nos permite ver con claridad la tensión que anida en las Leccioner.

${ }^{35}$ LFT, p. 168.

${ }^{36} \mathrm{DEHH}, \mathrm{p} .223$.

${ }^{37} \mathrm{DEHH}$, p. 223.

$38 \mathrm{DEHH}$, p. 124.

${ }^{39}$ DMS, p. 82.

40 Ángel Garrido-Maturano, "La encarnación del tiempo. Observaciones acerca de Ia relación entre tiempo y cuerpo a partir del pensamiento de E. Lévinas" en: 
Este autor advierte, en efecto, que por impresión originaria cabe entender, por un lado, el sentirse afectada la conciencia por una sensación puesto que el contenido de dicha impresión no es un producto de la conciencia sino algo ajeno a ella y frente a lo cual la conciencia comporta una recepción pasiva de un contenido nuevo, originario en un sentido eminente; por otro lado, sin embargo, y he aquí la oscuridad o tensión, "esta pasividad primera de la conciencia es también su espontaneidad primera, en tanto y en cuanto lo sentido por la conciencia es intuido como el material que constituye una fase del aparecer del objeto inmanente; es decir, de un objeto intencionado por una conciencia". Puesto en estos términos, el punto fuente con toda su originariedad es comprendido como parte de la dinámica de la conciencia, es decir, como el resultado de la libre intencionalidad de la conciencia. Consideramos aquí que Garrido Maturano tiene razón al preguntarse

si es la conciencia la que genera el presente y, consecuentemente el tiempo a partir de la Urimpression, o si, por el contario, una compresión radical de la noción husserliana de Urimpression (que, como el propio Husserl declara, supone uña génesis espontánea de la sensación) no nos conduce antes bien a pensar lo contrario, a saber: que es la conciencia la que se haya afectada por o padece el renovarse del presente corno constante paso de un tiempo que la excede, y cuya excedencia se manifiesta precisamente en lo imprevisible de cada sensación originaria ${ }^{41}$.

Encontramos este análisis de Garrido Maturano en un texto que recupera la lectura levinasiana de la Urimpression para subrayar su carácter innovador respecto del método fenomenológico en la medida que ella parece esbozar una inversión de dicho método al colocar entre paréntesis justamente el hecho de que se trata de una conciencia impresional (es decir, de un hecho de conciencia) para resaltar "su sentido originario vital", sentido que Lévinas mismo ubica en un nivel extranjero al pensamiento. En tal sentido, Garrido Maturano subraya el carácter altamente sugerente y alusivo

Ricardo de Souza, Andre de Farias y Marcelo Fabri (Organizadores), Alteridade e etica obra comemorativa dos 100 anos de nascimenio de Emmanuel Levinas, Porto Alegre, Edipucrs, 2008, pp. 77-101.

41 Ibid, p. 91. 
del siguiente pasaje levinasiano: "Ella [la Ur-impression] es por su parte colmada más allá de toda previsión, de toda espera, de todo germen y de toda continuidad, y, en consecuencia, es toda pasividad, receptividad de otro penetrando en el mismo, vida y no pensamiento"42.

La realización de la Urimpression fundamentalemente en el dominio de la vida no intencional, no pensante, permite a Garrido Maturano (lector de Lévinas) interpretar el proto-presente que comporta la sensación como un "presente diacrónico" anterior al presente de la representación. La distancia entre un tipo de presente y otro no es espacial sino temporal. Pero el "presente diacrónico" y el "presente de la representación" no marcan dos fracciones temporales diferentes de un mismo tiempo sino precisamente dos modalidades distintas del tiempo, inconmensurables; a saber: una, el tiempo del cuerpo; otra, el tiempo de la conciencia. Este autor señala el atributo irrepresentable de toda sensación y denomina a esa condición lo "sensacional" constitutivo de la sensación y el aspecto originario que reseña el prefijo Ur del término Urimpression.

\section{Observaciones finales.}

Observa Bernet que Lévinas va a proceder con Husserl como lo hizo con Heidegger: siendo más husserliano que Husserl pero leyendo a Husserl contra Husserl"43, es decir, colocando "la impresión originaria contra la representación intencional"44.

La interpretación levinasiana del concepto husserliano de Ur-impression introduce un matiz en esta noción subrayando la inicial pasividad con la que la conciencia se relaciona constantemente con cada nueva sensación.

\footnotetext{
42 DEHH, p. 216.
}

43 Fue Miguel Abensour (Cfr. Le mal elemental, 1991, pp. 28-29) quien acuñó la expresión "Heidegger contra Heidegger" para hacer referencia a la lectura de Lévinas de Ser y tiempo. En el contexto del artículo mencionado, a propósito específicamente del aprovechamiento antiheideggeriano de la hermenéutica de la facticidad. Rudolf Bernet advierte una lógica de lectura similar a la que el lituano lleva sobre la obra heideggeriana pero esta vez sobre textos acerca de la fenomenología del tiempo en Husserl.

${ }^{44}$ Ibidem, p. 151. 
Hallamos entonces una impugnación de la preeminencia de la intencionalidad de la representación por parte de la originariedad de la sensación, la que exige ser pensada como el acaecimiento de una realidad imprevisible y exterior a la conciencia.

Este sentido renovado de la Urimpression constituye un antecedente fenomenológico central para el proyecto levinasiano de la desformalización de la concepción del tiempo, pues descubre en el orden de las sensaciones acontecimientos que implican una modalidad específica y concreta de acaecer con excepción al régimen extático del tiempo interno regido por las fases de la conciencia protencional y retencional especialmente. Dicho orden se puede precisar como el de las afecciones que nos producen las cualidades sensibles de los elementos con los que nos relacionamos de manera prístina antes de que el pensamiento haga devenir dichas cualidades sin soporte en atributos de una sustancia.

Aquello que afecta en cada sensación, emerge, extraño al movimiento productivo de la conciencia, como una cualidad sensible exhibiendo la materialidad pura del elemento del que gozamos y del que nos alimentamos previo a tematizar los términos de esta relación. Antes e independientemente de la objetivación del contenido de la sensación, tiene lugar la pasión propia del padecimiento de la sensación que en el instante de su cumplimiento se realiza sin perspectivas de actividad percipiente. En este sentido, el padecimiento inherente a toda afección sensible expone el carácter de alteridad de lo otro; vale decir, de los elementos con los que nos vinculamos en el contacto físico.

La afección sensible de lo otro tiene como correlato puntual el presente de la Urimpression al que hemos caracterizado en el parágiafo anterior como "presente diacrónico" distinto al presente de la representación en tanto y en cuanto se produce de manera refractaria al movimiento sincrónico del tiempo interno de la conciencia. El presente originario, en el que constantemente se renuevan las protoimpresiones y sus correlativos puntos-ahora, es diacrónico porque se produce como un padecimiento constante de lo sensible revelando el origen foráneo de toda impresión o sensación respecto de la vida de la conciencia y evidenciando un modo propio de acaecer irreductible al presente de la representación. Ciertamente, es posible llevar el contenido de una conciencia retencional $\mathfrak{a}$ 
una representación, pero el componente "sensacional" de la sensación, que es el concreto padecimiento de ésta, tiene lugar en el presente de la protoimpresión que en el instante de su cumplimiento escapa a la intencionalidad retencional y por ende a la reflexión. En suma, el brote siempre imprevisible de las sensaciones junto a sus consecuentes padecimientos como vértice y modalidad en la que se produce el presente originario a través de la renovación constante de las protoimpresiones, describe el carácter diacrónico de esta dimensión temporal. Diacronía del presente absoluto o viviente de la Urimpression que interrumpe la hegemonía del régimen intencional y quiebra la continuidad del flujo inmanente del tiempo.

Cabe destacar que una de las consecuencias de la concepción del tiempo como flujo es que las dimensiones temporales del pasado y del futuro son concebidas por la fenomenología como actualizaciones del presente quedando la dialéctica interna de cada una de ellas reducida a "mera representación": representación del pasado y representación del futuro ${ }^{45}$. A contracorriente de esta concepción del pasado y del futuro como modificaciones del presente, la fenomenología del tiempo desarrollada por Lévinas intentará aportarles a estas dimensiones un espesor material, absolutamente independiente de la intencionalidad retencional, que dé cuenta por derecho propio - más acá y más allá del presente (retenido) - del acontecimiento del pasado y del futuro. La trascendencia de estos acontecimientos respecto de la intencionalidad retencional señala un nuevo orden temporal, por supuesto, refractario al de la conciencia - que Lévinas denomina, como dijimos, "diacronía" y que introduce precisamente ese instante de ruptura en el flujo. Las figuras de "pasado inmemorial" y las de "futuro puro" indicarán acontecimientos concretos que determinarán lo propio del pasado y del futuro por el sentido de su materialidad, oponiéndose a la idealidad de un pasado y un futuro proyectados desde el presente de la representación y anudados en el decurso continuo del tiempo interno. Del mismo modo, el presente viviente de la Urimpression exhibe una fecundidad fenomenoalógica -que Lévinas caracteriza como temporalización diacrónica- que excede la capacidad del presente de la representación.

${ }^{45}$ Futuro y pasado pertenecientes al tiempo extático de la conciencia que Lévinas mienta en $T A$ como "futuro del presente" y "pasado del presente". Al respecto, ver TA, pp. 63-64. 
Resta el saldo de aludir, en otro momento, a los términos en los que Lévinas sistematiza su propuesta de desformalización de la concepción del tiempo siguiendo el camino andado hasta Autrement qu'être, es decir, indicando de qué modo las distintas afecciones tienen como correlato otras dimensiones del tiempo diacrónico, a saber: "pasado anárquico" y "futuro puro".

\title{
Resumen
}

El presente trabajo se propone dar cuenta del sentido genérico de la noción levinasiana de diacronía y del influjo de la fenomenología del tiempo husserliana en la construcción de esa categoria. Inicialmente nos centramos en el texto de 1949, Le temps et l'Autre, en el que Lévinas presenta la figura de diacronía, centrándonos especialmente en su descripción como tiempo en el que se cumple la relación con la trascendencia. En segundo lugar, abordamos de manera más extensa el recorrido de la lectura levinasiana de las Lecciones de fenomenología de la conciencia del tiempo interno de Husserl, para sugerir ciertos nexos existentes entre la interpretación crítica de la noción de Urimpression y la de presente viviente husserlianas y su comprensión del tiempo diacrónico como un acontecimiento concreto anclado en la existencia sensible del sujeto, anárquica respecto dei presente de la representación.

Palabras clave: tiempo, trascendencia, alteridad, Urimpression, sensibilidad.

\begin{abstract}
This work intends to give reason of the generic sense of Levinas' notion of diachrony and of the influence of Husserl's phenomenology of time in the construction of that category. First, we concentrate ourselves in the 1949 text, Le temps et l'Autre, in which Lévinas introduces the figure of diachrony, and especially in his description of it as a time in which the relation with transcendence is fulfilled. The second thing we deal with more extensively, is the itinerary of the levinasian interpretation of Husserl's On the phenomenology of the consciousness of internal time, in order to suggest certain existing connections between the critical interpretation of the husserlian notions of Urimpression and of living present and the comprehension of diachronic time as a concrete event anchored in the subject's sensitive existence, which is anarchic as regards the present of representation.
\end{abstract}

Key words: time, trascendence, otherness, Urimpression, sensibility. 